\title{
Salivary biomarkers in patients with end-stage chronic kidney disease
}

\author{
Anna Nenova-Nogalcheva ${ }^{1}$, Assya Krasteva ${ }^{2}$, \\ Kamen Nogalchev ${ }^{3}$.
}

1. Departament of Oral Surgery, Faculty of Dental medicine, Medical University - Varna;

2. Department of oral and imaging diagnostics, Faculty of Dental medicine, Medical University Sofia;

3. Department of Prosthetic Dentistry, Faculty of Dental medicine, Medical University - Varna;

\section{Abstract}

Chronic kidney disease is defined as a progressive and irreversible deterioration of the renal function, with a rising incidence rate on a global scale. A wide range of oral changes can be observed in patients with end-stage renal disease, ones that reflect the changes occurring in harder and softer oral tissues, often as a result of the general manifestations of the basic condition. The objective of this review is to discuss the changes in the biological contents of the oral cavity with an emphasis on the most commonly used biological parameters consistent with the characteristics of chronic kidney diseases.

Keywords: saliva, diagnostic medium, chronic kidney disease, haemodialysis treatment, creatinine, urea 


\section{Background}

Chronic kidney disease (CKD) is a condition with significant social impact, which poses a serious health and social problem, affecting patients' lives. It is marked by a progressive loss of the renal function, and kidney damage over a period of months or years. Typically, the glomerular filtration rate is reduced and the serum creatinine and urea levels are increased. The most common causes contributing to this condition are diabetes mellitus, chronic glomerulonephritis, chronic interstitial nephrites, arterial hypertension, and autoimmune conditions $(1,2)$.

About $8 \%$ of the human population (over $550 \mathrm{mln}$. people) are affected by the disease. CKD incidence is approximately 120 people per million population. The rate of disease in Bulgaria is nearly $10 \%$ (about 700 000-750 000 people). In 2015, the total number of patients treated in dialysis centres across the country was 4,000 people. Of those, $90 \%$ are with terminal kidney failure and are on substitutive dialysis treatment. According to epidemiological data, the disease incidence, increases with age. Men are affected more often than women. Research has been made into the incidence of disease relative to ethnicity. In the US, according to written sources, mortality from CKD in the Asian race is lower than in the white Caucasian race $(2,3)$.

The questions linked to clarifying oral, dental and bone changes in patients with CKD, who are undergoing substitutive CHD (chronic haemodialysis) treatment are the subject of scientific research and developments but there are still unresolved issues in this regard. There are not many reports in medical literature regarding the use of salivary biomarkers as a diagnostic instrument in the evaluation of various systemic conditions, or for monitoring patients undergoing chronic haemodialysis.

\section{Review Results}

Chronic kidney disease can affect a wide range of tissues and systems. It directly or indirectly affects flow, concentrations and composition of saliva. In this review we would like to present the salivary biomarkers in patients with CKD.

\section{Salivary concentrations of $\mathrm{Na}, \mathrm{P}, \mathrm{K}, \mathrm{Ca}$ and urea}

Bella Ram Anuradha and coworkers have studied non-stimulated saliva from 50 patients with CKD undergoing substitutive treatment and found that the examined levels of $\mathrm{Na}, \mathrm{P}, \mathrm{K}, \mathrm{Ca}$, and urea in the saliva were considerably higher in patients with CKD, compared to the control group of healthy individuals. They found that the heightened levels in patients undergoing substitutive treatment, correlated to the severity of the kidney disease (4).

\section{Salivary concentrations of creatinine and urea}

In 2016, Taye Jemilat Lasisi et al. reported on research that proved that the salivary levels of creatinine and urea in patients with CKD reflected their levels in the blood. The authors concluded that salivary concentrations can be used as diagnostic biomarkers in chronic kidney disease, and can take prevalence over serum tests. The positive correlation between blood and saliva tests for creatinine and urea, 
observed in this research are consistent with previous reports by Marsh WH et al., Akai T, et al., Shannon IL et al. $(5,6)$.

\section{Salivary concentrations of urea, creatinine, $\mathrm{Na}, \mathrm{K}$, and phosphate}

C. Seethalakshmi and associates conducted a comparative study of patients with end- stage CKD for urea, creatinine, $\mathrm{Na}, \mathrm{K}$, and phosphate values in both serum and saliva, before and after substitutive treatment. Lower values were recorded for the tested indices in blood and saliva analysed before substitutive treatment, compared to the same, tested after it was conducted (7).

\section{Salivary concentrations of urea and nitrates (NO2-)}

In 2008, Blicharz TM and associates tested nitrates NO2- and urea in saliva samples taken from patients with end-stage kidney disease. The average salivary concentrations of the indices they analysed, were shown to go down with the length of dialysis, by $86 \%$ (nitrates) and $39 \%$ (urea). The authors concluded that salivary biomarker measurment can be an efficient alternative means of monitoring haemodialysis effectiveness (8).

\section{Salivary concentrations of urea and potassium}

Rafael M. Nagler and associates found in their research a very high correlation ratio between serum tested and saliva tested urea and potassium, and also recommended the use of salivary urea as a marker in the evaluation of dialysis treatment (9).

\section{Salivary concentrations of urea}

Suresh G. et al. also proved a correlation between the levels of urea in saliva and blood in patients undergoing haemodialysis as a result of CKD. The authors reached the conclusion that salivary tests for urea can replace the conventional blood tests (with priority particularly for children on substitutive treatment) (10).

\section{Conclusion}

All of the above data confirm the extraordinary opportunity in using the biological contents of the oral cavity instead of the conventional blood tests in the process of monitoring disease activity in patients with kidney damage. The undisputed advantages of saliva as diagnostic medium are well-known - an easily accessible testing material, non-invasive collection of samples that could be performed by the patient without the required participation of medical personnel, minimal infection risk, low cost value of the test (in laboratories routinely testing saliva). With the advance of laboratory methods, the biological contents in the oral cavity will be an increasingly preferred diagnostic medium. 


\section{References}

1.Asaumi J, Aiga $\mathrm{H}$, Hisatomi $\mathrm{M}$, et al. Advanced imaging in renal osteodystrophy of the oral and maxillofacial region. Dentomaxillofac Radiol 2001;30: 59-62.

2. Proctor R, Kumar N, Stein A, et al. Oral and dental aspects of chronic renal failure. J Dent Res 2005:84:199-208.

3. Cleber Souza, Ana Paula Braosi, Sonia Luczyszyn. Oral health in Brazilian patients with chronic renal disease. Med.Chili v.136 n.6 2008 741-746.

4. Beela Ram Anuradha, Sudheer Katta, Venkata Satyanarayana Kode et al. Oral and salivary changes in patients with chronic kidney disease: 2015 May-Jun; 19(3): 297-301.

5. Lasisi TJ, Raji YR, Salako BL. Salivary creatinine and urea analysis in patients with chronic kidney disease: a case control study. BMC Nephrol 2016; 16:17-10.

6. Kaushik A, Reddy SS, Umesh L, et al. Oral and salivary changes among renal patients undergoing haemodialysis: A cross-sectional study. Indian J Nephrol. 2013 Mar; 23(2):125-9.

7. Seethalakshmi C, Koteeswaran D, Chiranjeevi V. Correlation of Serum and Salivary Biochemical Parameters in end Stage Renal Disease Patients Undergoing Haemodialysis in Pre and Post-Dialysis State. J Clin Diagn Res. 2014 Dec;8(12):CC12-4.

8. Blicharz TM, Rissin DM, Bowden M, et al. Use of colorimetric test strips for monitoring the effect of haemodialysis on salivary nitrite and uric acid in patients with end-stage renal disease: a proof of principle. Clin Chem 2008; 54:1473-1480.

9. Nagler RM. Saliva analysis for monitoring dialysis and renal function. Clin Chem 2008; 54(9):1415-17.

10. Suresh G, Ravi Kiran A, Samata Y, et al. Analysis of Blood and Salivary Urea Levels in Patients Undergoing Haemodialysis and Kidney Transplant, J Clin Diagn Res. 2014; 8(7): 18-20.

\section{Corresponding author:}

Anna Nenova-Nogalcheva

Department of Oral Surgery, Faculty of Dental medicine, Medical University - Varna

anenova@yahoo.com 